\title{
Two-Level Proportional Hazards Models
}

\author{
Jerry J. Maples, ${ }^{1, *}$ Susan A. Murphy, ${ }^{2}$ and William G. Axinn ${ }^{3}$ \\ ${ }^{1}$ The Methodology Center and Department of Statistics, Pennsylvania State University, \\ 326 Thomas Building, University Park, Pennsylvania 16802, U.S.A. \\ ${ }^{2}$ Department of Statistics and Institute for Social Research, University of Michigan, \\ 4092 Frieze Building, Ann Arbor, Michigan 48109-1285, U.S.A. \\ ${ }^{3}$ Department of Sociology and Institute for Social Research, University of Michigan, \\ ISR-4046, 426 Thompson Street, Ann Arbor, Michigan 48106-1248, U.S.A. \\ *email: maples@stat.psu.edu
}

\begin{abstract}
SUmmary. We extend the proportional hazards model to a two-level model with a random intercept term and random coefficients. The parameters in the multilevel model are estimated by a combination of EM and Newton-Raphson algorithms. Even for samples of 50 groups, this method produces estimators of the fixed effects coefficients that are approximately unbiased and normally distributed. Two different methods, observed information and profile likelihood information, will be used to estimate the standard errors. This work is motivated by the goal of understanding the determinants of contraceptive use among Nepalese women in the Chitwan Valley Family Study (Axinn, Barber, and Ghimire, 1997). We utilize a two-level hazard model to examine how education and access to education for children covary with the initiation of permanent contraceptive use.
\end{abstract}

KEY wORDS: EM algorithm; Frailty model; Hazard model; Multilevel; Profile likelihood; Random coefficient; Semiparametric likelihood; Survival analysis.

\section{Introduction}

Grouped data occurs in a wide variety of applications, e.g., in smoking cessation trials where individuals are in support groups, in educational trials where children are grouped into schools, in community-based studies where individuals are grouped into neighborhoods, and in genetic studies in which individuais are grouped into families. In many of these same applications, the response is the time until an event, such as the time until relapse, the time until marijuana use initiation, time until use of permanent methods of contraception, or time until appearance of a disease. Consequently, there has been considerable interest in survival analysis models for grouped or multilevel data (e.g., see Clayton, 1978; Oakes, 1982; McGilchrist and Aisbett, 1991; Yashin, Vaupel, and Iachine, 1995; Sastry, 1997; Sinha and Dey, 1997; Vaida and $\mathrm{Xu}, 2000$ ). Statisticians have recognized that two individuals within the same group will have responses that are more similar than two individuals in two different groups. This increased similarity in responses may be conceptualized as due to a shared group-level covariate (Kreft, Leeuw, and Aiken, 1994).

For example, in the Chitwan Valley Family Study (CVFS), neighborhoods of women are sampled in Chitwan Valley, Nepal (Axinn, Barber, and Ghimire, 1997; Axinn and Barber, 2001). A primary goal of this study is to understand the determinants of changing fertility patterns. Of particular interest is the association between a woman's schooling and the timing, relative to the birth of their first child, of initiation of a permanent method of contraception. Some women initiate contraceptive use quickly, averting potential births, while others initiate use late, after bearing many children. Education is expected to increase the opportunity costs of childbearing, motivating more rapid initiation of contraceptive use. The multiple links between the spread of education and contraceptive behavior, including both neighborhood-level effects of schools and individual-level effects of schooling, motivate multilevel models of the variation in contraceptive use timing (Axinn, 1993; Axinn and Barber, 2001). Additionally, although research has made great advances in the measurement of all neighborhood information that may correlate with the timing of initiation of permanent contraceptive use (Axinn et al., 1997), it is clear that women in the same neighborhood are likely to have more similar timing patterns than women across neighborhoods.

To allow dependency of response times within a group, shared frailty models were developed (Vaupel, Manton, and Stallard, 1979; Clayton and Cuzick, 1985; Oakes, 1989; Guo and Rodriguez, 1992; Klein, 1992). The shared frailty model is a multilevel extension of the proportional hazards model (Cox, 1972) whereby a frailty (random intercept) term, which varies from group to group, is introduced in the regression model. In effect, different groups experience the event at proportionately different baseline rates. In the shared frailty model, within-group comparisons of hazard rates at different 
levels of an individual-level covariate must be the same across groups. In the CVFS, researchers hypothesized that women who have received formal education initiated a permanent method of contraception at a higher rate than women without formal education (Axinn and Barber, 2001). Furthermore, it is plausible that, in some neighborhoods, there may be little difference in initiation rates between women of different education levels and, in other neighborhoods, women of different educational levels may vary greatly in their initiation rate. Thus, to consider this type of question, we extend the shared frailty model to allow for random coefficients.

This article will discuss a two-level proportional hazards model that incorporates random variability in the baseline rate and random coefficients for an individual-level covariate. Next, we derive the likelihood and elucidate the assumptions behind the likelihood. Parameter estimates will be based on maximum likelihood using a combination of the EM algorithm and Newton-Raphson. Standard errors will be estimated using two different methods, observed information and profile likelihood information. A simulation study will follow to empirically demonstrate properties of the model. The CVFS data will be used to illustrate the applicability of this two-level hazard model. Last, advantages, disadvantages, and areas in need of further research will be discussed.

\section{Two-Level Hazard Model}

\subsection{Hazard Model}

We formulate a two-level hazard model for continuous event time data; this model will allow a nonparametric specification of the baseline hazard function. First, the hazard model will be developed and then the likelihood will be given along with a statement of assumptions. Finally, estimation procedures for point estimates and standard errors will be presented.

Let $T_{i j}$ be the event time for the $j$ th individual $(j=$ $\left.1, \ldots, n_{i}\right)$ nested in the $i$ th group $(i=1, \ldots, N)$, where the event times are measured with enough precision to assume there are no ties. Furthermore, let $Z_{i}(t)$ denote a vector of group-level covariates at time $t, X_{i j}(t)$ denote a $p$-dimensional vector of individual-level covariates at time $t$, and $R_{i}=$ $\left(R_{i 0}, R_{i 1}\right)$ denote $(p+1)$-dimensional vector of unobserved group-level random effects. For a study of duration $M$, on each individual, we observe the minimum of the event time, $T_{i j}$, and the censoring time, $C_{i j}$, and an indicator $\Delta_{i j}$, where $\Delta_{i j}=1$ if $T_{i j}<C_{i j}$ and 0 otherwise. Thus, for group $i$, we observe $\left(T_{i j} \wedge C_{i j}, \Delta_{i j},\left\{X_{i j}(t), t \in\left[0, T_{i j} \wedge C_{i j}\right]\right\}\right.$, $\left.j=1, \ldots, n_{i},\left\{Z_{i}(t), t \in[0, M]\right\}\right)$, and the groups are assumed to be a random sample from the population.

Note that the above $Z$ is assumed to be measured up to time $M$. In this article, we consider only group-level covariates (Z's) that are measured independently of the sampled members of the group. Thus, the group-level covariate exists independently from the individuals of the group and, although $Z$-values will not be used after the last person in the group experiences the event or is censored, the group values do exist.

To facilitate the analysis (in Section 4) of the timing of initiation of a permanent method of contraception in the CVFS data, we require that the hazard model incorporates the following multilevel features. First, the hazard model should allow static and time-varying covariates on both the individual and group levels. Additionally, the model should accommodate interactions between the individual- and group- level covariates. Second, the hazard model should allow the effect of individual-level covariates to vary between groups. Third, the baseline hazard rates should be permitted to vary between the groups. Finally, if there is no systematic variation in the hazard rates between the groups after accounting for the observed covariates, the model should reduce to Cox's proportional hazard model.

We assume a proportional hazards model for $T_{i}$ given $R_{i}$ of

$$
\begin{aligned}
& \lim _{d t \rightarrow 0} d t^{-1} P\left[t \leq T_{i j}<t+d t \mid X_{i j}(s), Z_{i}(s), s \leq t, T_{i j} \geq t, R_{i}\right] \\
& \quad=\lambda_{0}(t) \exp \left\{R_{i 0}+R_{i 1}^{\mathrm{T}} X_{i j}(t)+\beta_{x}^{\mathrm{T}} X_{i j}(t)+\beta_{z}^{\mathrm{T}} Z_{i}(t)\right\} \\
& \quad=h_{i j}(t)
\end{aligned}
$$

where $\lambda_{0}(t)$ is the baseline hazard function. Because the above hazard is conditional on the random effects, the $\beta_{x}$ coefficients reflect a comparison of responses within the same or identical groups. The variance of $R_{i 0}$ measures the heterogeneity of the baseline rate between the groups. The variance of $R_{i 1}$ measures the heterogeneity in the coefficient of the individual-level covariates $X_{i j}$ between the groups. We assume that the marginal distribution of the random effects is multivariate normal with mean zero and arbitrary covariance matrix $\Sigma$. If $\Sigma$ is zero, then the responses of individuals within a group are independent and this model reduces to the proportional hazards model. We use normally distributed random effects for two reasons. The normal distribution family is closed under linear combinations and matches our view that unexplained variation in hazard rates between groups is due to a large number of unobserved group-level covariates.

In addition to the shared frailty model, other multilevel survival models include Hedeker and Gibbons' (1996) development of a multilevel hazard model for interval-time survival data and Barber et al.'s (2000) development of a multilevel hazard model for discrete-time survival data. Yashin et al. (1995) developed a correlated frailty model to study the role of genetics versus environmental factors in influencing individual mortality. Sastry (1997) developed a three-level shared frailty model to study the mortality of children in Brazil. Gilks et al. (1993), Sinha and Dey (1997), Gustafson (1997), and Sargent (1998) have taken a Bayesian approach to modeling grouped survival data.

Vaida and $\mathrm{Xu}(2000)$ also consider the proportional hazards model with random effects in the hazard function

$$
h_{i j}(t)=\lambda_{0}(t) \exp \left(\beta^{\prime} X_{i j}^{*}+W_{i j}^{\prime} R_{i}\right),
$$

where $X_{i j}^{*}$ 's are the group- and individual-level covariates and $W_{i j}$ is the covariate vector for the random effects, $R_{i}$. There are two main differences between the hazard models in (1) and (2). First, in our hazard model (1), $W_{i j}=$ $\left(1, X_{i j}\right)$, while Vaida and Xu's model allows for a more general random effects structure. Second, our model allows timevarying covariates on both the individual- and group-level covariates. As in our model, the vector of random effects, $R_{i}$, in (2) is assumed to follow a multivariate normal distribution with mean zero and unknown covariance matrix. Vaida and $\mathrm{Xu}$ use an EM algorithm with MCMC integration in the Estep to estimate the parameters and they use Louis's (1982) 
formula to obtain the observed information matrix. Our article provides an alternative procedure for point estimation and computing standard errors and makes the underlying assumptions explicit.

\subsection{Likelihood}

We follow the likelihood derivation of Nielsen et al. (1992) to provide a partial likelihood for the parameters, $\beta=$ $\left(\beta_{x}, \beta_{z}\right)$ (the fixed effects), $\Sigma$ (covariance matrix of random effects), and $\Lambda_{0}=\int_{0}^{t} \lambda_{0}(s) d s$ (the cumulative baseline hazard function). The likelihood derivation and assumptions can be made rigorous by using counting process terminology (Aalen, 1976) and the methods developed by Andersen et al. (1993) and Arjas and Haara (1984). Our primary purpose is to provide an intuitive explanation of the assumptions that are made in addition to classical survival analysis assumptions.

We begin by considering one group only. To keep the notation simple, omit the subscript $i$, denoting group. Also let $Y_{j}$ denote the observed minimum of the event and censoring time for an individual, $Y_{j}=T_{j} \wedge C_{j}$. Thus, the observations for a group are $\left(Y_{j}, \delta_{j},\left\{X_{j}(t), t \in\left[0, T_{j} \wedge C_{j}\right]\right\}, j=1, \ldots, n\right.$, $\left.\left\{Z(t), t \in\left[0, \max _{j} Y_{j}\right]\right\}\right)$.

First, we make a conditional independence assumption. Let $\mathcal{F}_{t-}=\left\{R, T_{j} I\left\{T_{j}<t\right\}, I\left\{T_{j}<t\right\},\left\{X_{j}(s), s \in\right.\right.$ $\left.\left.\left.\left[0, T_{j} \wedge t\right)\right\}, j=1, \ldots, n,\left\{Z(s), s \in\left[0, \max _{j} T_{j} \wedge t\right)\right\}\right)\right\}$, where $I\{A\}$ is one if the event $A$ is true and zero otherwise. This conditional independence assumption may be expressed in terms of conditional probabilities given $\mathcal{F}_{t \ldots}$. We assume that, for each $j$,

$$
P\left[t \leq T_{j}<t+d t \mid \mathcal{F}_{t-}\right] \approx h_{j}(t) I\left\{T_{j} \geq t\right\} d t
$$

for small $d t$ and where $h_{j}(t)=\lambda_{0}(t) \exp \left\{R_{0}+R_{1}^{\mathrm{T}} X_{j}(t)+\right.$ $\left.\beta_{x}^{\mathrm{T}} X_{j}(t)+\beta_{z}^{\mathrm{T}} Z(t)\right\}$, as in the previous section. Thus, conditional on $R$, the hazard of $T_{j}$ remains the same whether or not we include information on the other subjects in the group up to time $t$. This assumption is the analog of the conditional independence assumption made in multilevel linear and nonlinear models, i.e., given the random effects, responses within a group are assumed independent (e.g., see the conditional likelihoods in Rodriguez and Goldman (1995, equation (7)) or Hedeker and Gibbons (1994, equation (2))). Note that our parameterization of $h_{j}$ involves only the $j$ th individual's covariates and not the covariates of other individuals in the group. Thus, if we believe that individual $j$ 's covariate, given by $X_{j}$, is predictive of the $j$ th individual's response, then $X_{j^{\prime}}$ needs to be included in the $j$ th individual's covariate vector.

Following the likelihood derivation given by Nielsen et al. (1992) for shared frailty model, we assume

(3) conditional on $R=r$, censoring is independent,

(4) conditional on $R=r$, censoring is noninformative of $r$, and

(5) conditional on $R=r$, the covariates are noninformative of $r$

These three assumptions plus the conditional independence assumption (3) and the conditional proportional hazards assumption (1) imply that the group's contribution to a partial likelihood for $\left(\beta, \Sigma, \Lambda_{0}\right)$ is

$L\left(\beta, \Sigma, \Lambda_{0}\right)$

$$
\begin{aligned}
\int_{\mathcal{R}^{p+1}} & {\left[\prod_{j=1}^{n} h_{j}\left(Y_{j}\right)^{\delta_{j}} \exp \left\{-\int_{0}^{Y_{j}} h_{j}(s) d s\right\}\right] } \\
& \times(2 \pi)^{-(p+1) / 2}|\Sigma|^{-1 / 2} \exp \left\{-\frac{1}{2} \mathbf{r}^{T} \Sigma^{-1} \mathbf{r}\right\} d \mathbf{r} .
\end{aligned}
$$

To form the partial likelihood for the $N$ groups, we subscript $L\left(\beta, \Sigma, \Lambda_{0}\right)$ by $i$ to denote the contribution by group $i$ and multiply across groups to get

$$
L\left(\beta, \Sigma, \Lambda_{0}\right)=\prod_{i=1}^{N} L_{i}\left(\beta, \Sigma, \Lambda_{0}\right) .
$$

Assumption (3), independent censoring, is commonly made in the estimation of single-level hazard models and has been discussed by many authors (Kalbfleisch and Prentice, 1980; Andersen et al., 1988; Liang, Self, and Chang, 1993). To extend this work to the multilevel setting, we make the additional assumptions (4) and (5). Assumptions (4) and (5) are surprisingly stringent. They concern the conditional distribution given the past of the censoring process and the covariate process, respectively. To illustrate the stringent nature of these assumptions, we focus on assumption (5). If all of the covariates are time independent, then assumption (5) is simply that the covariates are independent of $R$. Marginal independence between the covariates and the random effects is commonly made in multilevel analyses (Bryk and Raudenbush, 1992; Guo and Rodriguez, 1992; Hedeker and Gibbons, 1996). Define $\mathcal{F}_{t-}^{\text {obs }}$ to be $\mathcal{F}_{t-}$ but adjusting for the loss of information due to censoring, i.e., $\mathcal{F}_{t-}^{\text {obs }}=$ $\left\{R, Y_{j} I\left\{Y_{j}<t\right\}, \delta_{j} I\left\{Y_{j}<t\right\}, I\left\{Y_{j}<t\right\},\left\{X_{j}(s), s \in\right.\right.$ $\left.\left.\left.\left[0, Y_{j} \wedge t\right)\right\}, j=1, \ldots, n,\left\{Z(s), s \in\left[0, \max _{j} Y_{j} \wedge t\right)\right\}\right)\right\}$ Suppose all time-dependent covariates are exogenous in the sense that the conditional proportional hazards assumption, conditional independence assumption, and assumptions (3) and (4) continue to hold even if we include the entire history of the covariate over the interval $[0, M]$ in $\mathcal{F}_{t-}, \mathcal{F}_{t-}^{\text {obs }}$. Then, as before, assumption (5) is simply that the covariates are marginally independent of $R$ (we have included the entire history of the covariate over the interval $[0, M]$ in $\mathcal{F}_{t-}, \mathcal{F}_{t-}^{\text {obs }}$ ),

Assumption (5) is most stringent when the time-dependent covariates do not satisfy the above exogeneity conditions e.g., marginal independence of $R$ and $X$ does not imply that assumption (5) is satisfied. This is because assumption (5) requires conditional independence of $R$ and $X(t)$ given past observations on $T$. In order to ensure that (5) is a reasonable assumption, we should try to include all common correlates of both the $T_{j}$ 's and the covariates in our model, and similarly, in order to ensure that $(4)$ is a reasonable assumption, we should try to include all common correlates of both the $T_{j}$ 's and the censoring times. In the Appendix, we illustrate how marginal independence of $R$ and $X$ is insufficient for assumption (5) to hold.

\subsection{Estimation}

To estimate the parameters, we maximize an empirical version of the partial likelihood as in Nielsen et al. (1992) and Murphy and van der Vaart (2000). We replace the $\lambda_{0}(t)$ terms by jumps in the cumulative hazard, $\Delta \Lambda_{0}(t)=\Lambda_{0}(t)-\Lambda_{0}(t-)$. The empirical version of the partial likelihood is then

$L\left(\beta, \Sigma, \Lambda_{0}\right)$ 


$$
\begin{aligned}
=\prod_{i=1}^{N} \int_{\mathcal{R}^{p+1}} & {\left[\prod_{j=1}^{n_{i}}\left(\Delta \Lambda_{0}\left(Y_{i j}\right) e^{g_{i j}\left(Y_{i j}\right)}\right)^{\delta_{i j}}\right.} \\
& \left.\times \exp \left\{-\int_{0}^{Y_{i j}} e^{g_{i j}(s)} d \Lambda_{0}(s) d s\right\}\right] \\
& \times(2 \pi)^{-(p+1) / 2}|\Sigma|^{-1 / 2} \exp \left\{-\frac{1}{2} \mathbf{r}^{\mathrm{T}} \Sigma^{-1} \mathbf{r}\right\} d \mathbf{r}
\end{aligned}
$$

where $Y_{i j}=T_{i j} \wedge C_{i j}$ and $g_{i j}(t)=r_{i 0}+r_{i 1}^{\mathrm{T}} X_{i j}(t)+\beta_{\mathcal{X}}^{\mathrm{T}} X_{i j}(t)+$ $\beta_{z}^{\mathbf{T}} Z_{i}(t)$. We will maximize (4) over all nondecreasing $\Lambda_{0}$, all positive definite $\Sigma$, and real-valued vectors $\beta_{x}$ and $\beta_{z}$. For any fixed value of $(\beta, \Sigma)$, the above partial likelihood will be maximized for $\Lambda_{0}(t)$, a nondecreasing function in $t$ with positive jumps at the observed event times.

The partial likelihood (4) is maximized over $\left(\beta, \Sigma, \Lambda_{0}\right)$ by using a combination of EM (Dempster, Laird, and Rubin, 1977) and Newton-Raphson. By using both EM and NewtonRaphson methods, the weaknesses of either algorithm are greatly diminished. We use the EM algorithm to first move close to a maximum and then switch to Newton-Raphson to take advantage of its quicker convergence rate. In general, maximizing (3) by EM requires the estimation of the conditional expectations $\mathrm{E}\left[r_{i} r_{i}^{\mathrm{T}} \mid y, \tilde{\theta}\right]$ and $\mathrm{E}\left[e^{r_{i 0}+X_{i j} r_{i 1}} \mid y, \tilde{\theta}\right]$, where $\tilde{\theta}$ 's are the current estimates of $\left(\beta, \Lambda_{0}, \Sigma\right)$. We estimate these integrals using a 13-point Gauss-Hermite quadrature. Vaida and $\mathrm{Xu}$ (2000) also use the EM algorithm, but instead of using a Gauss-Hermite quadrature, they use MCMC to approximate the integrals. Once the expectations are formed, the M-step for $\beta$ follows the usual $\log$ partial likelihood in the Cox model with known offsets and $\Delta \Lambda_{0}(t)$ is estimated by

$$
\Delta \Lambda(t)=\left\{\sum_{y_{i j} \geq t} \mathrm{E}\left[e^{g_{i j}\left(y_{i j}\right)} \mid y, \tilde{\theta}\right]\right\}^{-1}
$$

\subsection{Standard Errors}

Standard errors can be estimated by at least two different methods, observed information and profile likelihood information. The estimated observed information is the negative of the Hessian matrix,

$$
\hat{I}_{\mathrm{obs}}=-\frac{\partial^{2}}{\partial \theta \theta^{\mathrm{T}}} \log L(\hat{\theta})
$$

where $\theta=\left(\beta, \Lambda_{0}, \Sigma\right)$. This matrix is used by the NewtonRaphson algorithm for parameter estimation. The asymptotic covariance matrix is then obtained by inverting $\hat{I}_{\text {obs }}$. Because the dimension of the observed information matrix is greater than the number of observed events in the data, it can be quite large.

An alternative method to estimate the standard errors is to use the observed profile likelihood information as in Murphy and van der Vaart (1996). A profile likelihood for $\beta$ is

$$
\operatorname{prlik}(\beta)=\max _{\Lambda_{0}, \Sigma} \log L(\theta) .
$$

This is treating $\Lambda_{0}$ and $\Sigma$ as nuisance parameters with respect to inference concerning $\beta$. An estimator of the asymptotic covariance matrix of $\hat{\beta}$ is obtained by inverting the observed profile information matrix,

$$
\hat{I}_{\text {prof }}=-\frac{\partial^{2}}{\partial \beta^{\prime} \beta^{T}} \operatorname{prlik}(\hat{\beta}) .
$$

In a similar fashion, we can form a profile likelihood for each component in $\Sigma$ and then use the observed profile information matrix to form estimators of the asymptotic variance for the estimators of the components in $\Sigma$. Patefield (1977) has shown that the standard errors based on the full likelihood and the profile likelihood are equivalent for the reduced parameter space in parametric models. Although the profile likelihood method does not have an explicit expression, we approximate the second derivative through a combined forward and backward finite difference (Murphy and van der Vaart, 1996).

The maximum likelihood estimates and their standard errors (from either the profile likelihood information or the observed information) can be used to create $z$-statistics. In the next section, we assess how well the distribution of the $z$-statistics can be approximated by a normal distribution.

\section{Simulations}

In this section, a simulation study is used to assess the performance of the point estimators and standard errors. Because the primary use of the estimated standard errors is to construct a $z$-statistic or confidence interval, the accuracy of the standard errors is assessed indirectly by examining the coverage level of confidence intervals based on the estimated standard errors. All of the simulations use the hazard model $h_{i j}(t)=\lambda_{0}(t) \exp \left\{R_{i 0}+\left(\beta+R_{i 1}\right) X_{i j}\right\}$, where $X_{i j}$ is a single individual-level static covariate. We consider parameter values that lead not only to nonproportionality but also crossing of the marginal hazard rates.

Each simulation consists of 1000 generated datasets of 50 groups, with each group having uniformly random 410 subjects. We consider two covariate distributions, a Bernoulli(.5) and a skewed distribution- $X=\mathrm{E}-1$, where $E \sim$ exponential(1). The failure-time distribution is a Weibull,

$$
f(t)=p t^{p-1} e^{r_{0}+\left(\beta+r_{1}\right) x} \exp \left\{-t^{p} e^{r_{0}+\left(\beta+r_{1}\right) X}\right\} .
$$

We use two values of $p: p=1$ (flat baseline hazard, $\lambda(t)=1$ ) or $p=1.5$ (increasing baseline hazard, $\lambda(t)=t^{1 / 2}$ ). For each group, the values for the random effects are drawn from a bivariate normal $(0, \Sigma)$. The variance-covariance matrix, $\Sigma$, will have equal entries on the diagonal. Two censoring levels, 10 and $20 \%$, are considered. The event times are censored at the 90 th or 80 th percentile of the event-times distribution conditional on the covariate.

Table 1 gives the parameters values for all six representative simulations. For each of the 1000 simulated datasets, $95 \%$ confidence intervals were created for each parameter using the profile likelihood information standard errors. The mean, empirical $95 \%$ confidence interval, and coverage probability are listed in Table 2.

Simulations 1-4 represent a variety of situations in which we expect the point estimates and estimated standard errors to perform well. Because each simulation consists of 1000 datasets, we expect the coverage percentages to be between 93.6 and $96.4 \%$. Only simulation 4 yields a coverage percent for $\beta$ that falls outside of this interval. In this case, increasing 
Table 1

Simulated datasets ${ }^{\mathrm{a}}$

\begin{tabular}{|c|c|c|c|c|c|c|}
\hline Sim. & $\beta$ & $\begin{array}{c}\text { Censor } \\
\text { rate } \\
(\%)\end{array}$ & $\begin{array}{l}\text { Hazard } \\
\text { shape }\end{array}$ & $\begin{array}{l}\text { Var. } \\
\text { RE }\end{array}$ & Corr. & Covariate \\
\hline 1 & 1 & 10 & Flat & .1827 & 0 & Bernoulli \\
\hline 2 & 1 & 10 & Flat & 1 & 0 & Bernoulli \\
\hline 3 & .25 & 20 & Increasing & .4112 & .5 & Bernoulli \\
\hline 4 & -0.25 & 20 & Increasing & .4112 & -.5 & Exponential \\
\hline 5 & 1 & 10 & Flat & 0 & 0 & Bernoulli \\
\hline 6 & 1 & 10 & Flat & .1827 & 0 & Bernoulli \\
\hline
\end{tabular}

${ }^{a}$ Parameter values for the simulation study. In simulation 6 , the random effects come from a demeaned gamma distribution rather than a normal distribution. Simulation 2 has converging hazard functions. Simulations 3 and 4 have crossing hazard functions.

the sample size to 100 yields a coverage percent falling in the interval. Because all of the 50-group simulations had a coverage slightly less than $95 \%$ and the point estimators appear to be unbiased, this may be an indication that, in small samples, the profile likelihood information slightly underestimates the standard error for $\beta$. In all four simulations, the standard deviations of the random effects are underestimated. The downward bias was typically larger for the random coefficient than the random intercept. This bias can be expected because the estimators do not take into account the loss in degrees of freedom from the estimation of $\beta$ (Fahrmeir and Tutz, 1994, Chapter 7).

For simulations 5 and 6 , the assumptions behind the model are violated and we thus examine the robustness of the model. In simulation $5, \Sigma=\mathbf{0}$, the variance matrix for the random effects, is on the boundary of the parameter space and thus we do not expect the point estimates and estimated standard errors to behave well. In simulation 6 , the setup is again. identical to simulation 1 except that the random effects are generated from independent demeaned gamma distribution $W-1$, where $W$ has mean 1 and variance .1827 . Simulation 5 showed the most problems, which was to be expected. However, the estimator of $\beta$ had only a slight positive bias and the approximate $95 \%$ confidence intervals performed well. Unlike the negative bias exhibited by the estimated standard deviations in simulations $1-4$ and 6 , the estimated standard deviations are positively biased in simulation 5 . This positive bias is expected because the estimation algorithm forces the parameter estimates to stay within the interior of the parameter space. With the exception of the covariance term, the point estimates and estimated standard errors performed well in simulation 6 , even when the random effects distribution was misspecified.

Table 2

Simulation results

\begin{tabular}{|c|c|c|c|c|c|c|}
\hline Sim. & $\begin{array}{l}\text { No. } \\
\text { groups }\end{array}$ & Parameter & $\begin{array}{c}\text { True } \\
\text { value }\end{array}$ & $\begin{array}{l}\text { Mean } \\
\text { value }\end{array}$ & $\begin{array}{c}95 \% \\
\text { Conf, int. }\end{array}$ & $\begin{array}{c}\text { Coverage } \\
\text { percent }\end{array}$ \\
\hline \multirow[t]{4}{*}{1} & 50 & $\beta$ & 1 & 1.007 & $(.997,1.016)$ & .946 \\
\hline & & $\mathrm{SD}$ (int.) & .427 & .409 & $(.401, .418)$ & .947 \\
\hline & & $\operatorname{cov}$ & 0 & -.008 & $(-.015,-.002)$ & .986 \\
\hline & & $\mathrm{SD}$ (coef.) & .427 & .395 & $(.382, .407)$ & .943 \\
\hline \multirow[t]{4}{*}{2} & 50 & $\beta$ & 1 & .999 & $(.987,1.012)$ & .939 \\
\hline & & $\mathrm{SD}$ (int.) & 1 & .970 & $(.959, .980)$ & .937 \\
\hline & & $\mathrm{cov}$ & 0 & .023 & $(.007, .039)$ & .938 \\
\hline & & $\mathrm{SD}$ (coef.) & 1 & .947 & $(.934, .961)$ & .954 \\
\hline \multirow[t]{4}{*}{3} & 50 & $\beta$ & .25 & .253 & $(.242, .263)$ & .941 \\
\hline & & $\mathrm{SD}$ (int.) & .641 & .629 & $(.621, .638)$ & .961 \\
\hline & & $\operatorname{cov}$ & .206 & .185 & $(.186, .204)$ & .982 \\
\hline & & $\mathrm{SD}$ (coef.) & .641 & .615 & $(.602, .629)$ & .919 \\
\hline \multirow[t]{4}{*}{4} & 50 & $\beta$ & -.25 & -.242 & $(-.251,-.235)$ & .925 \\
\hline & & $\mathrm{SD}$ (int.) & .641 & .620 & $(.613, .627)$ & .980 \\
\hline & & cov & -.205 & -.202 & $(-.209,-.195)$ & .966 \\
\hline & & $\mathrm{SD}$ (coef.) & .641 & .611 & $(.602, .620)$ & .919 \\
\hline \multirow[t]{4}{*}{$4(\mathrm{a})$} & 100 & $\beta$ & -.25 & -.249 & $(-.256,-.242)$ & .953 \\
\hline & & $\mathrm{SD}$ (int.) & .641 & .627 & $(.620, .633)$ & .925 \\
\hline & & $\operatorname{cov}$ & -.205 & -.199 & $(-.207,-.191)$ & .923 \\
\hline & & $\mathrm{SD}$ (coef.) & .641 & .618 & $(.608, .627)$ & .983 \\
\hline \multirow[t]{4}{*}{5} & 50 & $\beta$ & 1 & 1.017 & $(1.008,1.026)$ & .945 \\
\hline & & $\mathrm{SD}$ (int.) & 0 & .155 & $(.147, .162)$ & .913 \\
\hline & & $\operatorname{cov}$ & 0 & -.004 & $(-.005,-.003)$ & .992 \\
\hline & & $\mathrm{SD}$ (coef.) & 0 & .231 & $(.221, .242)$ & .869 \\
\hline \multirow[t]{4}{*}{6} & 50 & $\beta$ & 1 & .996 & $(.987,1.006)$ & .948 \\
\hline & & $\mathrm{SD}$ (int.) & .427 & .410 & $(.401, .419)$ & .938 \\
\hline & & $\mathrm{cov}$ & 0 & -.018 & $(-.025,-.011)$ & .988 \\
\hline & & $\mathrm{SD}$ (coef.) & .427 & .400 & $(.387, .413)$ & .935 \\
\hline
\end{tabular}


Table 3

Comparison of standard errors for $\beta$

\begin{tabular}{lcccc}
\hline \hline & $\begin{array}{c}\text { True } \\
\text { value }\end{array}$ & $\begin{array}{c}\text { Coverage } \\
\text { (prof. like.) }\end{array}$ & $\begin{array}{c}\text { Coverage } \\
\text { (obs. info. }\end{array}$ & $\begin{array}{c}\text { Number of } \\
\text { datasets obs. } \\
\text { info. problems }\end{array}$ \\
\hline 1 & 1 & .946 & .946 & 7 \\
2 & 1 & .939 & .938 & 6 \\
3 & .25 & .941 & .942 & 17 \\
4 & -.25 & .925 & .920 & 2 \\
$4(\mathrm{a})$ & -.25 & .953 & .953 & 3 \\
5 & 1 & .945 & .947 & 29 \\
6 & 1 & .948 & .946 & 10 \\
\hline
\end{tabular}

${ }^{a}$ Datasets in which the Hessian matrix used in calculating the observed information was not positive definite.

In Table 3, a comparison between the two standard error estimation methods, profile likelihood information and observed information, is made. We focus on the estimated standard errors for $\beta$ and compare the coverage of the $95 \%$ normal-theory confidence intervals. The two methods have nearly identical coverage properties; however, in some of the datasets where the parameter estimates for the covariance matrix of the random effects were on the boundary of the parameter space, the Hessian matrix was not positive definite.

\section{Chitwan Valley Family Study}

The Chitwan Valley Family Study (CVFS) was designed to measure dynamic changes in socioeconomic context from a sample of 171 neighborhoods in south-central Nepal and to link these changes to individual-level life histories for the purpose of explaining marriage timing, childbearing, and contraceptive use (Axinn et al., 1997). Neighborhoods are defined as clusters of 5-15 households, which fits the settlement pattern in Chitwan (Axinn et al., 1997). The retrospective histories of change in each neighborhood were collected with the aid of the neighborhood history calendar method (Axinn et al., 1997). In each sampled neighborhood, CVFS interviewed every resident aged 15-59. Residents were asked to provide complete life histories of childbearing, contraceptive use, education, and related behaviors. Building on the life history calendar method (Freedman et al., 1988), the investigators developed an advanced form of the life history calendar, including memory cues from the neighborhood history data (Axinn, Pearce, and Ghimire, 1999).

A central aim of the CVFS was the examination of the timing of initiation of permanent contraceptive use. The vast majority of contraception in Nepal is used for stopping childbearing rather than spacing births. For the purposes of this example, we consider initiation of the use of intrauterine device (IUD), Norplant, and depo-provera to be permanent methods (see Axinn and Barber (2001) for a more complete list and discussion). Because permanent contraceptive use among women who have no children is extremely rare in this setting, we will only estimate rates of initiation of permanent contraceptive use for women who have given birth to at least one child. The time of first use of a permanent method of contraception is measured in months from the birth of a woman's first child. If a woman had not used contraceptives at the time of the interview, then the censoring time was measured in months from the birth of the woman's first child to the interview.

We use this study to illustrate the usefulness of the proposed methodology. More complete multilevel analyses can be found in Axinn and Barber (2001) and Axinn and Yabiku (2001). Because this particular substantive application is designed as an illustration of the multilevel model, we do not provide a comprehensive examination of other potential methodological issues relevant to the substantive subject, such as problems of retrospective recall (see Diamond, McDonald, and Shah, 1986) or bias due to differential death rates.

Access to schooling for children, the level of education of the woman, and birth cohort are among the factors that may predict the rate at which women start to use contraceptives. The main hypotheses that will be tested here are as follows:

Hypothesis 1. Do women with some formal education have a higher rate of initiating a permanent method of contraception compared with uneducated women within their neighborhood?

Hypothesis 2. Does the effect of women's education on the time of initiation of a permanent method of contraception vary across the neighborhoods?

Hypothesis 3. Do women who are located in neighborhoods closer to schools have a higher rate of initiating a permanent method of contraception compared with women in neighborhoods further away from schools?

We considered a sample of 81 neighborhoods, all within 9.7 miles of the Narayanghat, the major town in Chitwan Valley. Within the neighborhoods, we considered all women between the ages of 25 and 44 (born between 1952 and 1971) at the time of the 1996 interview, each of whom had given birth to at least one child. This sample is composed of 488 ever-married women with a range of 1-15 women per neighborhood.

In the simple model below, we use three covariates. The indicator (COHORT) is equal to one for the older women, who were between the ages of 35 and 44 during the interview (born between 1952 and 1961), and equal to zero for the younger women (born between 1962 and 1971). If the woman had any formal education prior to the birth of her first child, then the indicator for education, (EDU), equaled one. In the older cohort of women, 55 ont of $196(28.1 \%)$ had received any formal education prior to the birth of their first child. For the younger cohort of women, 160 out of $292(54.8 \%)$ had received any formal education. To measure access to educational opportunities for children, the indicator (DIST-SCH) was equal to one if if there was a school within a 5-minute walk of the neighborhood and zero otherwise. The distance to the nearest school is a time-varying covariate at the neighborhood level. Prior to the 1950s, public schools were extremely rare in the Chitwan Valley area, but since, there has been a dramatic increase in the number of schools, thus increasing the educational opportunities for children.

Assumptions (3) and (4) from Section 2.2 are trivially satisfied as censoring occurs at the number of months corresponding to the length of time from the birth of the woman's first child to the time of the interview. Consider assumption (5). The only time-dependent covariate, distance to the nearest school, is a neighborhood-level variable. Because decisions concerning school placement were made by the central government, we believe that this covariate is exogenous in 
Table 4

Results from analysis of CVFS

\begin{tabular}{lrrc}
\hline \hline & Estimate & $\mathrm{SE}$ & $Z$-score $(p$-value $)$ \\
\hline Covariates & & & \\
$\quad$ Education & .579 & .0461 & $12.56(<.0001)$ \\
Cohort & -.121 & .0473 & $2.55(.0108)$ \\
Edu cohort & -.488 & .0456 & $10.70(<.0001)$ \\
$\quad$ Dist school & .193 & .0372 & $5.18(<.0001)$ \\
Variance component & & & \\
SD $\left(r_{0 i}\right)$ & .571 & .128 & $4.46(<.0001)$ \\
SD $\left(r_{1 i}\right)$ & .381 & .297 & $1.44(.2005)$ \\
Covariance & -.132 & .169 & $0.781(.4348)$ \\
\hline
\end{tabular}

the sense that the distance from a neighborhood to a school is independent of past actions by women in the neighborhood. Thus, conceptually, we may include the entire history of the distance-to-nearest-school covariate in $\mathcal{F}_{t-}, \mathcal{F}_{t}^{\text {obs }}$; i.e., in terms of forming the likelihood, we treat distance to the nearest school as a time-independent covariate. So assumption (5) reduces to assuming that the random effect $R$ is marginally independent of all of the covariates.

In order to address the three hypotheses, we fit the following hazard model:

$$
\begin{aligned}
h_{i j}(t)=\lambda_{0}(t) \exp \{ & R_{0 i}+\left(\beta_{1}+R_{1 i}\right) \mathrm{EDU}_{i j}+\beta_{2} \mathrm{COHORT}_{i} \\
& +\beta_{3} \mathrm{EDU}-\mathrm{COHORT}_{i j} \\
& \left.+\beta_{4} \mathrm{DIST}-\mathrm{SCH}_{i}(t)\right\} .
\end{aligned}
$$

The parameter estimates and standard errors are presented in Table 4. All of the covariates were significant in the model. From the model and Table 4, we can address the three main hypotheses for this analysis. Because the interaction between birth cohort and the woman's education was significant, we must address hypothesis 1 for each cohort separately. In the younger cohort, the women with some formal education prior to the birth of their first child had a hazard rate $e^{\hat{\beta}_{1}}=e^{.579}=$ 1.78 times higher than women without any education ( $p$-value $<.0001$ ). However, for the older cohort of women, the hazard rate was $e^{\hat{\beta}_{1}+\hat{\beta}_{3}}=e^{.091}=1.09\left(\operatorname{SE}\left(\hat{\beta}_{1}+\hat{\beta}_{3}\right)=.059, p\right.$ value $=.1231$ ) times higher than the older women without any formal education, but this was not a significant difference. For a more complete discussion, including consideration of the availability of contraceptive methods and the relation between education and the use of permanent contraceptives, see Axinn and Barber (2001).

The variance for the random coefficient $\left(R_{1 i}\right)$ for women's education, used to test the second hypothesis is nonsignificant $(p$-value $=.2005)$, providing evidence that the covariation of education with initiation of a method of permanent contraception is constant across neighborhoods. From the third hypothesis, women who lived in neighborhoods with schools nearby, within a 5-minute walk, had a $21 \%$ higher hazard rate $\left(e^{\hat{\beta}_{4}}=e^{.193}=1.21\right)$ for initiation than women in neighborhoods that did not have a school nearby $(p$-value $<.0001)$. In addition to the main hypotheses, the model also gives further insight into the data. The variance of the baseline hazard rate between the neighborhoods, $\operatorname{var}\left(R_{0 i}\right)$, is significant ( $p$-value $<$

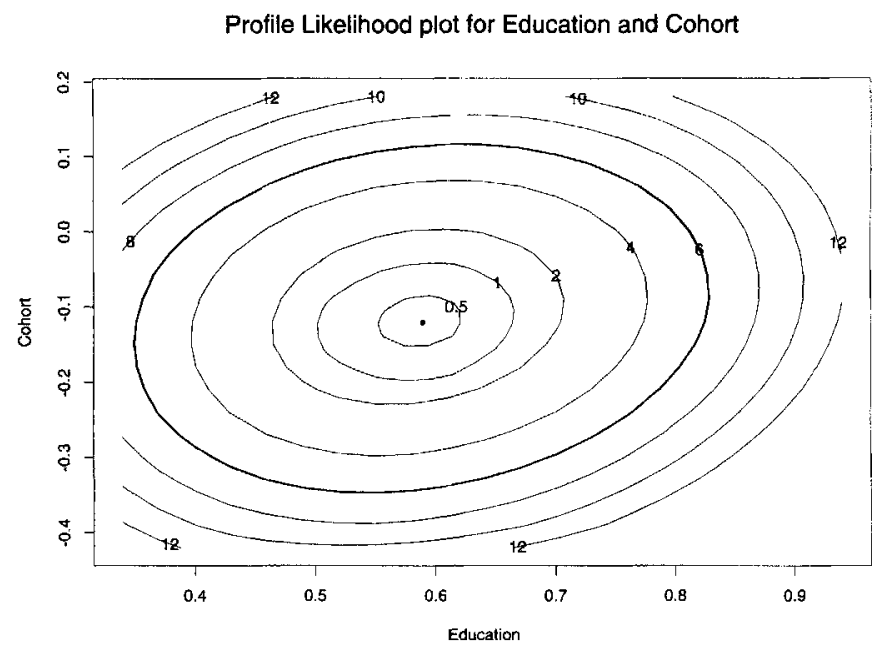

Figure 1. Profile likelihood surface for parameters $\beta_{1}$ (education) and $\beta_{2}$ (cohort). The contour lines represent two times the difference in likelihood value from the maximum likelihood value at the MLE. Assuming normality, the darkened contour line of 6 represents a $95 \%$ joint confidence region for $\beta_{1}$ and $\beta_{2}$.

.0001). We will discuss credibility of these inferences, in particular the credibility of the estimated standard errors, using profile likelihood surface plots.

Examining the profile likelihood surface for a pair of parameters can show how well the normal-theory $Z$-tests are working. If the normality approximation holds, then the contours of the profile likelihood surface will be elliptical. The profile likelihood surface plot for $\left(\beta_{1}, \beta_{2}\right)$, the effects of woman's education and cohort, is shown in Figure 1. The contours represent twice the difference in profile likelihood value from the maximum likelihood. The elliptical shape of the contour lines indicates that the standard errors should work well, i.e., the $95 \%$ confidence set for $\left(\beta_{1}, \beta_{2}\right)$ is quadratic. The profile likelihood surface plot for $\left(\mathrm{SD}\left(R_{0}\right), \operatorname{SD}\left(R_{1}\right)\right)$ is shown in Figure 2. In this plot, the contour lines do not have the nice elliptical shapes as before. Viewing the plot from the $y$-axis, it is clear that the quadratic approximation of the likelihood does not hold for estimation of the standard deviation of the random coefficient; however, viewing the plot from the $x$-axis, we see that the surface of the profiled likelihood does appear quadratic in the standard error of the random intercept. Certainly some skepticism is in order in interpreting the $p$-values for the variance components.

\section{Discussion}

The two-level proportional hazards model allows for heterogeneity between groups as well as for effects of individuallevel covariates to vary by group, i.e., random coefficients. The fixed effects represent comparisons of individuals within the same or highly similar groups rather than a comparison between individuals from a variety of neighborhoods. In contrast to the frailty model, this model allows us to examine the level of covariation between a subject-level covariate such as woman's education and neighborhood. A combination of 
Profile Likelihood piot for Std. Dev of random effects

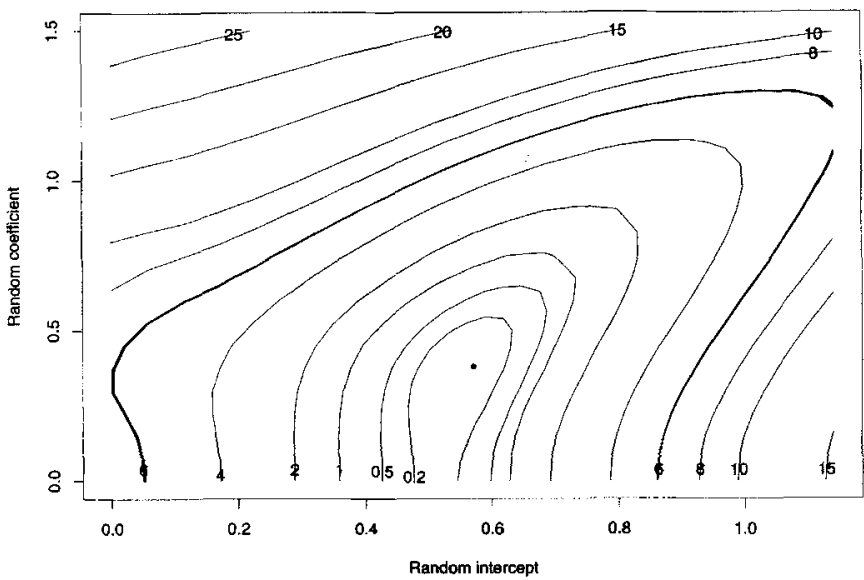

Figure 2. Profile likelihood surface for standard deviations of $r_{0}$ (random intercept) and $r_{1}$ (random coefficient). The contour lines represent two times the difference in likelihood value from maximum likelihood value at the MLE. Assuming normality, the darkened contour line of 6 represents a $95 \%$ joint confidence region for the standard deviations of $r_{0}$ and $r_{1}$.

EM and Newton-Raphson algorithms are used to obtain parameter estimates. Standard errors may be estimated using the observed information or a finite difference of the profile likelihood.

When the fixed effect, $\beta$, is of primary scientific interest, the simulations in Section 3 provide evidence for the usefulness of this model and estimation method for datasets as small as 50 groups. The estimators and standard errors for the variance components need improvement, especially the estimator of the covariance term. It is easy to see how the model can be extended to accommodate any number of random coefficients and more complicated random effects design. However, computational issues in point estimation and standard errors must be carefully considered. The development of methods other than the traditional EM and Newton-Raphson are needed for these high-dimensional maximization problems. Alternatives include both Vaida and Xu's (2000) use of MCMC integration and Raudenbush, Yang, and Yosef's (2000) use of a multivariate Laplace approximation to the likelihood function. Further, asymptotic theory for the estimators in this model still needs to be developed. Finally, formal testing of the variance of the random effects being equal to zero needs to be explored, as this is often an important research hypothesis.

\section{ACKNOWLEDGEMENTS}

This research was supported by grant 1P50-DA-10075 from NIDA to the Pennsylvania State University's Methodology Center, grant HD32912 from NICHD, NSF grants SBR 9811983 and DMS 9802885, and a P30 center grant from NICHD to the Institute for Social Research at the University of Michigan.

\section{RÉSUMÉ}

Nous proposons une extension du modèle de taux proportionnels à un modèle à deux niveaux avec un terme con- stant aléatoire et des coefficients aléatoires. Les paramètres de ce modèle à plusieurs niveaux sont estimés par une combinaison des algorithmes EM et de Newton-Raphson. Même pour des échantillons de 50 groupes, la méthode fournit pour les coefficients des effets fixes, des estimateurs à peu près sans biais et normalement distribués. Deux méthodes distinctes, information observée et information de la vraisemblance des profils, seront utilisées pour estimer les erreurs standards. Ce travail a été entrepris dans le but de comprendre les déterminants des habitudes contraceptives des femmes Népalèses dans l'enquête sur les familles de la Vallée Chitwan (Axinn, Barber et Ghimire, 1997). Nous avons utilisé ce modèle de taux proportionnels à deux niveaux pour étudier la façon dont le niveau d'éducation et les possibilités d'accès à l'éducation des enfants varient avec l'initiation à un usage permanent de la contraception.

\section{REFERENCES}

Aalen, O. (1976). Nonparametric inference in connection with multiple decrement models. Scandinavian Journal of Statistics 3, 15-27.

Andersen, P., Borgan, O., Gill, R., and Keiding, N. (1988). Censoring, truncation and filtering in statistical methods based on counting processes. Contemporary Mathematics 80, $19-60$.

Andersen, P., Borgan, O., Gill, R., and Keiding, N. (1993). Statistical Models Based on Counting Processes. New York: Springer-Verlag.

Arjas, E. and Haara, P. (1984). A marked point process approach to censored failure data with complicated covariates. Scandinavian Journal of Statistics 11, 193-209.

Axinn, W. (1993). The effects of children's schooling on fertility limitation. Population Studies 47, 481-493.

Axinn, W. and Barber, J. (2001). Mass education and fertility limitation. American Sociological Review 66, 481-505.

Axinn, W. and Yabiku, S. (2001). Social change, the social organization of families, and fertility limitation. American Journal of Sociology 106, 1219-1261.

Axinn, W., Barber, J., and Ghimire, D. (1997). Sociological methodology. In: The Neighborhood History Calendar, A. Raftery (ed.), 355-392. Oxford: Blackwell Publishers.

Axinn, W., Pearce, L., and Ghimire, D. (1999). Innovations in life history calendar applications. Social Science Research 28, 243-264.

Barber, J., Murphy, S., Axinn, W., and Maples, J. (2000). Discrete time multilevel hazards analysis. Sociological Methodology 30, 201-235.

Bryk, A. and Raudenbush, S. (1992). Hierarchical Linear Models. Thousand Oaks, California: Sage Publications.

Clayton, D. (1978). A model for association in bivariate life tables. Biometrika 65, 141-151.

Clayton, D. and Cuzick, J. (1985). Multivariate generalizations of the proportional hazards model. Journal of the Royal Statistical Society, Series A 148, 82-117.

Cox, D. (1972). Regression models and life tables. Journal of the Royal Statistical Society, Series B 34, 187-220.

Dempster, A., Laird, N., and Rubin, D. (1977). Maximum likelihood estimation from incomplete data via the EM algorithm. Journal of the Royal Statistical Society, Series $B$ 39, 1-38. 
Diamond, I. D., McDonald, J. W., and Shah, I. H. (1986). Proportional hazards models for current status data: Application to the study of differentials in age at weaning in Pakistan. Demography 23, 607-620.

Fahrmeir, L. and Tutz, G. (1994). Multivariate Statistical Modelling Based on Generalized Linear Models. New York: Springer-Verlag.

Freedman, D., Thornton, A., Camburn, D., Alwin, D., and Yong-DeMarco, L. (1988). The life history calendar: A technique for collecting retrospective data. In Sociological Methodology 1988, C. C. Clogg (ed.), 37-38. Washington, D.C.: American Sociological Association.

Gilks, W., Clayton, D., Spiegelhalter, D., Best, N., and McNeil, A. (1993). Modeling complexity: Applications of Gibbs sampling in medicine. Journal of the Royal Statistical Society, Series B 55, 39-52.

Guo, G. and Rodriguez, G. (1992). Estimating a multivariate proportional hazards model for clustered data using the EM algorithm. Journal of the American Statistical Association 87, 969-976.

Gustafson, P. (1997). Large hierarchical Bayesian analysis of multivariate survival data. Biometrics 53, 230-242.

Hedeker, D. and Gibbons, R. (1994). A random-effects ordinal regression model for multilevel analysis. Biometrics 50, 993-944.

Hedeker, D. and Gibbons, R. (1996). MIXOR: A computer program for mixed effects ordinal regression analysis. Computer Methods and Programs in Biomedicine 49, 157-176.

Kalbfleisch, J. and Prentice, R. (1980). The Statistical Analysis of Failure Time Data. New York: Wiley.

Klein, J. (1992). Semiparametric estimation of random effects using the Cox model based on the EM algorithm. Biometrics 48, 795-806.

Kreft, I., Leeuw, J, and Aiken, L. (1994). The effect of different forms of centering in hierarchical linear models. Technical Report 30. Research Triangle Park, North Carolina: National Institute of Statistical Sciences.

Liang, K., Self, S., and Chang, Y. (1993). Modeling marginal hazards in multivariate failure time data. Journal of the Royal Statistical Society, Series B 55, 441-453.

Louis, T. (1982). Finding observed information using the EM algorithm. Journal of the Royal Statistical Society, Series B 44, 98-130.

McGilchrist, C. and Aisbett, C. (1991). Regression with frailty in survival analysis. Biometrics 47, 461-466.

Murphy, S. and van der Vaart, A. (1996). Semiparametric likelihood ratio inference. Technical Report 96-03. University Park, Pennsylvania: Pennsylvania State University, Department of Statistics.

Murphy, S. and van der Vaart, A. (2000). On profile likelihood. Journal of the American Statistical Association 95, 449-485.

Nielsen, G., Gill, R., Andersen, P., and Sorensen, T. (1992). A counting process approach to maximum likelihood estimation in frailty models. Scandinavian Journal of Statistics 19, 25-43.

Oakes, D. (1982). A model for bivariate survival data. Journal of the Royal Statistical Society, Series B 44, 414-422.
Oakes, D. (1989). Bivariate survival models induced by frailties. Journal of the American Statistical Association 84, $487-493$.

Patefield, W. (1977). On the maximized likelihood function. Sankhya, Series B 39, 92-96.

Raudenbush, S., Yang, M., and Yosef, M. (2000). Maximum likelihood for generalized linear models with nested random effects via high-order, multivariate Laplace approximation. Journal of Computational and Graphical Statistics 9, 141-157.

Rodriguez, G. and Goldman, N. (1995). An assessment of estimation procedures for multilevel models with binary responses. Journal of the Royal Statistical Society, Series $A$ 158, 73-89.

Sargent, D. (1998). A general framework for random effects survival analysis in the Cox proportional hazards setting. Biometrics 54, 1486-1497.

Sastry, N. (1997). A nested frailty model for survival data. Journal of the American Statistical Association 92, 426435.

Sinha, D. and Dey, D. (1997). Semiparametric Bayesian analysis of survival data. Journal of the American Statistical Association 92, 1195-1212.

Vaida, F. and Xu, R. (2000). Proportional hazards model with random effects. Statistics in Medicine 19, 3309-3324.

Vaupel, J., Manton, K., and Stallard, E. (1979). The impact of heterogeneity in individual frailty on the dynamics of mortality. Demography 16, 439-454.

Yashin, A., Vaupel, J., and Iachine, I. (1995). Correlated individual frailty: An advantageous approach to survival analysis of bivariate data. Demography 34, 31 48 .

\section{Received June 2000. Revised April 2002.} Accepted April 2002.

\section{APPENDIX}

\section{Example for Assumption 5}

The following example illustrates how $R$ and $X$ may be marginally independent yet, given observation on $T, R$, and $X$, are dependent. There is no censoring. The covariate, $X(t)$, is identically zero (with probability one) for $t \leq t_{0}$ and is discrete valued thereafter. Furthermore, suppose that there is an unobserved binary common correlate, say $U$, of both $T$ and $X\left(t_{0}+d t\right)$. The joint density of $(R, U, S=I\{T \geq$ $\left.\left.t_{0}\right\}, X\left(t_{0}+d t\right)\right)$ evaluated at $(r, u, s, x)$ can be written as

$$
\begin{aligned}
& f_{R}(r) P[U=u] P\left[T \geq t_{0} \mid R=r, U=u\right]^{s} \\
& \quad \times P\left[T<t_{0} \mid R=r, U=u\right]^{1-s} P\left[X\left(t_{0}+d t\right)=x \mid U=u\right],
\end{aligned}
$$

where $f_{R}$ is the density of $R$. To be concrete, let $P[U=0]=$ $P[U=1]=1 / 2, P\left[T \geq t_{0} \mid R=r, U=1\right]=2 e^{-e^{r} t_{0}}-$ $e^{-2 e^{r} t_{0}}$, and $P\left[T \geq t_{0} \mid R=r, U=0\right]=e^{-2 e^{r} t_{0}}$. It is easy to see that $X$ is marginally independent of $R$ and $U$ is marginally independent of the random effect, $R$. The hazard model constrains the form of $P\left[T \geq t_{0} \mid R=r\right]=\Sigma_{u} P[U=u] \times$ 
$P\left[T \geq t_{0} \mid R=r, U=u\right]$ (in this case, $P\left[T \geq t_{0} \mid R=\right.$ $r]=e^{-e^{r} t_{0}}$ ) and specifies that $f_{R}$ is a normal distribution; the other assumptions impose no further constraints. Because both $U$ and $R$ are causes of $T$, we can expect that $U$ and $R$ are correlated given $T \geq t_{0}$, i.e.,

$$
P\left[U=0 \mid T \geq t_{0}, R=r\right]=\frac{1 / 2 e^{-2 e^{r} t_{0}}}{e^{-e^{r} t_{0}}}
$$

depends on $R=r$. However, $U$ is also correlated with $X\left(t_{0}+\right.$ $d t)$. Thus, we can expect that $X\left(t_{0}+d t\right)$ and $R$ are correlated given $T \geq t_{0}$, i.e.,

$$
\begin{aligned}
& P\left[X\left(t_{0}+d t\right)=x \mid T \geq t_{0}, R=r\right] \\
& \quad=\frac{1 / 2 e^{-2 e^{r} t_{0}} P\left[X\left(t_{0}+d t\right)=x \mid U=0\right]}{e^{-e^{r} t_{0}}} \\
& \quad+\frac{1 / 2\left(2 e^{-e^{r} t_{0}}-e^{-2 e^{r} t_{0}}\right) P\left[X\left(t_{0}+d t\right)=x \mid U=1\right]}{e^{-e^{r} t_{0}}}
\end{aligned}
$$

will generally depend on $R=r$. In this case, assumption (5) will be violated. 